\title{
Pituitary duplication and nasopharyngeal teratoma in a newborn: CT, MRI, US and correlative histopathological findings
}

Huisman, Thierry A G M ; Fischer, Ursin ; Boltshauser, Eugen ; Straube, Torsten ; Gysin, Claudine

\begin{abstract}
The computed tomography and MRI imaging findings in a case of pituitary duplication and epipharyngeal teratoma are described in a newborn baby girl with respiratory difficulties. Associated skull base and central nervous system malformations are presented. Teratoma diagnosis was confirmed by histology. The embryological pathogenesis is discussed
\end{abstract}

DOI: https://doi.org/10.1007/s00234-005-1374-8

Posted at the Zurich Open Repository and Archive, University of Zurich ZORA URL: https://doi.org/10.5167/uzh-156346

Journal Article

Published Version

Originally published at:

Huisman, Thierry A G M; Fischer, Ursin; Boltshauser, Eugen; Straube, Torsten; Gysin, Claudine (2005). Pituitary duplication and nasopharyngeal teratoma in a newborn: CT, MRI, US and correlative histopathological findings. Neuroradiology, 47(7):558-561.

DOI: https://doi.org/10.1007/s00234-005-1374-8 
Thierry A. G. M. Huisman

Ursin Fischer

Eugen Boltshauser

Torsten Straube

Claudine Gysin
Pituitary duplication and nasopharyngeal
teratoma in a newborn: CT, MRI, US
and correlative histopathological findings

Abstract The computed tomography and MRI imaging findings in a case of pituitary duplication and epipharyngeal teratoma are described in a newborn baby girl with respiratory difficulties. Associated skull base and central nervous system malformations are presented. Teratoma diagnosis was confirmed by histology. The embryological pathogenesis is discussed.
Keywords Pituitary duplication · Teratoma $\cdot$ Malformation
T. A. G. M. Huisman $(\bowtie) \cdot$ T. Straube Department of Diagnostic Imaging, University Children's Hospital Zurich, Steinwiesstrasse 75, 8032 Zurich, Switzerland

E-mail: thierry.huisman@kispi.unizh.ch

Tel.: +41-1-266-7110

Fax: +41-1-266-7158

U. Fischer - C. Gysin

Division of Ear, Nose and Throat Surgery,

University Children's Hospital Zurich,

Steinwiesstrasse 75, Zurich, Switzerland

E. Boltshauser

Department of Neurology,

University Children's Hospital Zurich,

Steinwiesstrasse 75, Zurich, Switzerland pharyngeal teratoma and a complex of associated midline malformations including pituitary gland duplication.

\section{Case report}

A baby girl, born after an uneventful pregnancy of 38 weeks, presented with respiratory distress on day 1 of life. Physical examination raised the suspicion of a nasopharyngeal obstruction. Endoscopic examination showed a soft tissue mass protruding from the nasopharynx into the mesopharynx through a partially cleft palate. The lesion presented as a hairy mass with areas that resembled hemangiomatous components as well as ectodermal components including epidermis. She had 
mildly dysmorphic facial features with hypertelorism. No other associated abnormalities were seen. Blood oxygenation levels were always within normal limits. Because of poor feeding, a gastric tube was inserted for feeding. Neurological examination was unremarkable. The child's biometrics were within normal limits. Family history was unremarkable.

MRI was performed on day 5 of life. A T1- and T2heterogeneous soft tissue mass with inclusion of fatty components and calcifications was seen within the roof of the epipharynx in tight connection to the sphenoid bone (Fig. 1). The maximal diameter was $2.8 \mathrm{~cm}$. The mass protruded through a partially cleft palate into the dorsal part of the mesopharynx. The choanes were partially obliterated by the mass. MRI also showed a complete duplication of the pituitary gland including the pituitary stalk. The mammillary bodies and the tuber cinereum were fused into a single midline mass resulting in a thickening of the third ventricle floor. The olfactory bulb was lacking on one side. In addition, a partial duplication of the distal basilar artery was seen. Hypertelorism was confirmed; eye globes and optic nerves were without pathology. The central nervous system was otherwise normal; specifically, no additional midline malformations were seen.
Computer tomography (CT) was added on day 9 of life for a precise preoperative evaluation of the osseous skull base. CT showed a bony cleft within the sphenoid (Fig. 2). A wide separation of the anterior clinoid processes was seen as well as a wide dorsal aspect of the bony nasal septum. The sella was separated into two distinct cavities containing both pituitary glands separated by a bony ridge. The epipharyngeal mass showed a mixture of hypodense fatty components (negative Hounsfield units) next to hyperdense bony and hypodense soft tissue contents indicative of a mature teratoma. The partially cleft palate was confirmed. Otherwise the skull base was unremarkable.

Ultrasonography of the abdomen did not show any additional malformations or midline defects. The bony skeleton including the entire spine was also without signs of fusion or segmentation anomalies.

The child was operated on 7 days later. The soft tissue mass could be resected without complications. Tumor histology confirmed the diagnosis of a mature teratoma (Fig. 3). No cerebrospinal fluid leakage occurred. The surgical reconstruction of the cleft palate is planned at 18 months of age. The child is thriving and developing well since operation.
Fig. 1 a Coronal T1-weighted precontrast and postcontrast spin echo ( $S E)$ (upper row) and T2-weighted fast SE (FSE) (lower row) magnetic resonance $(M R)$ images. A full duplication of the pituitary gland including the pituitary stalk and the infundiblum as well as a fusion of the mammillary bodies and the tuber cineurum with thickening of the floor of the third ventricle is seen. b Coronal T1-weighted SE (left) and T2-weighted FSE (center and right) MRI. T1hyperintense fat is seen within the nasophayrngeal teratoma (left). The coronal T2-weighted FSE image (center) reveals a normal left olfactory bulb, while the right olfactory bulb is lacking. In addition, a partial duplication of the distal basilary artery is observed (right). c Sagittal T2-weighted FSE MR image. A thickening of the floor of the third ventricle due to fusion of the mammillary bodies and the tuber cinereum is seen next to the heterogeneous partially $\mathrm{T} 2$ hyperintense and hypointense teratoma within the epipharynx adajcent to the sphenoid bone
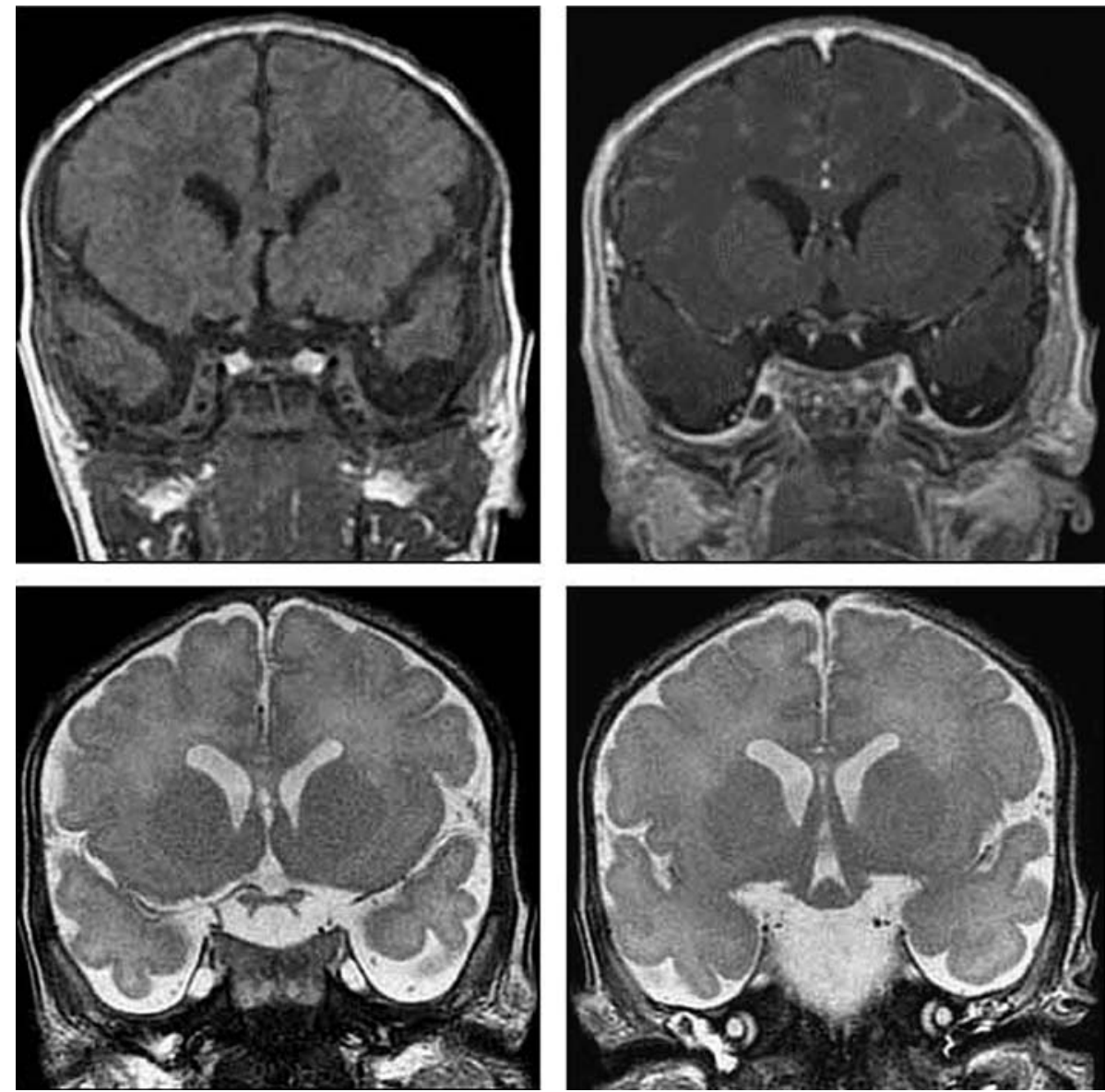

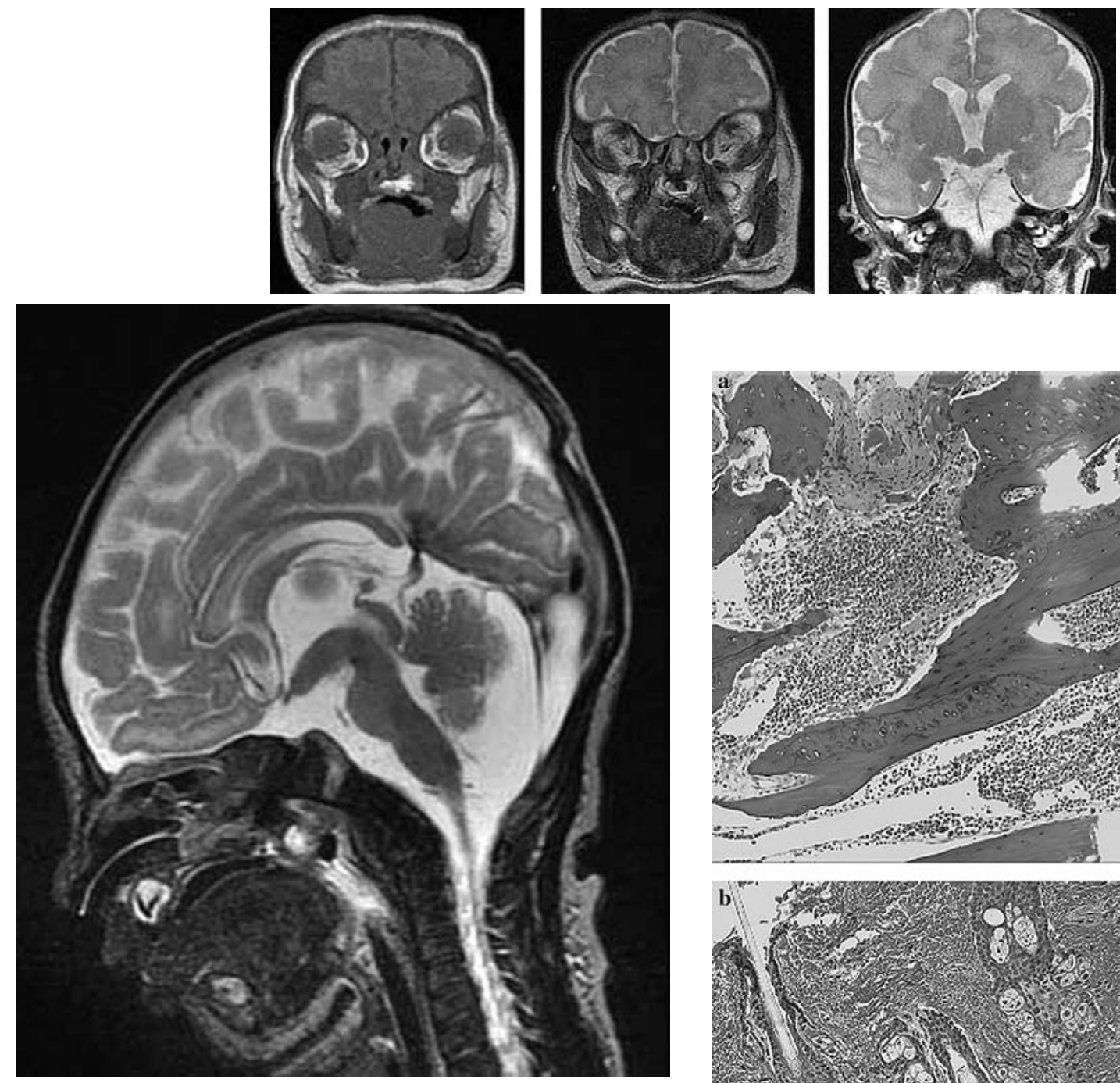

Fig. 1 (Contd.)
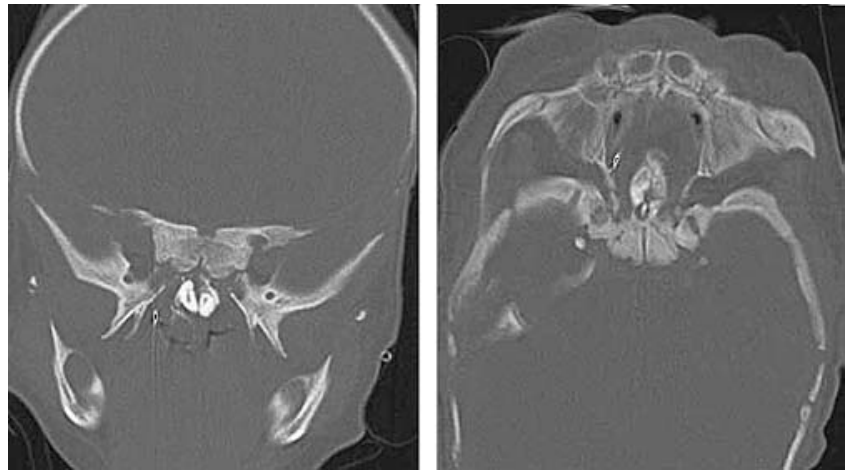

Fig. 2 Coronal and axial computer tomography (bone window setting). Hyperdense calcifications within the nasophayngeal teratoma as well as a sagittal cleft within the widened sphenoid bone is visualized
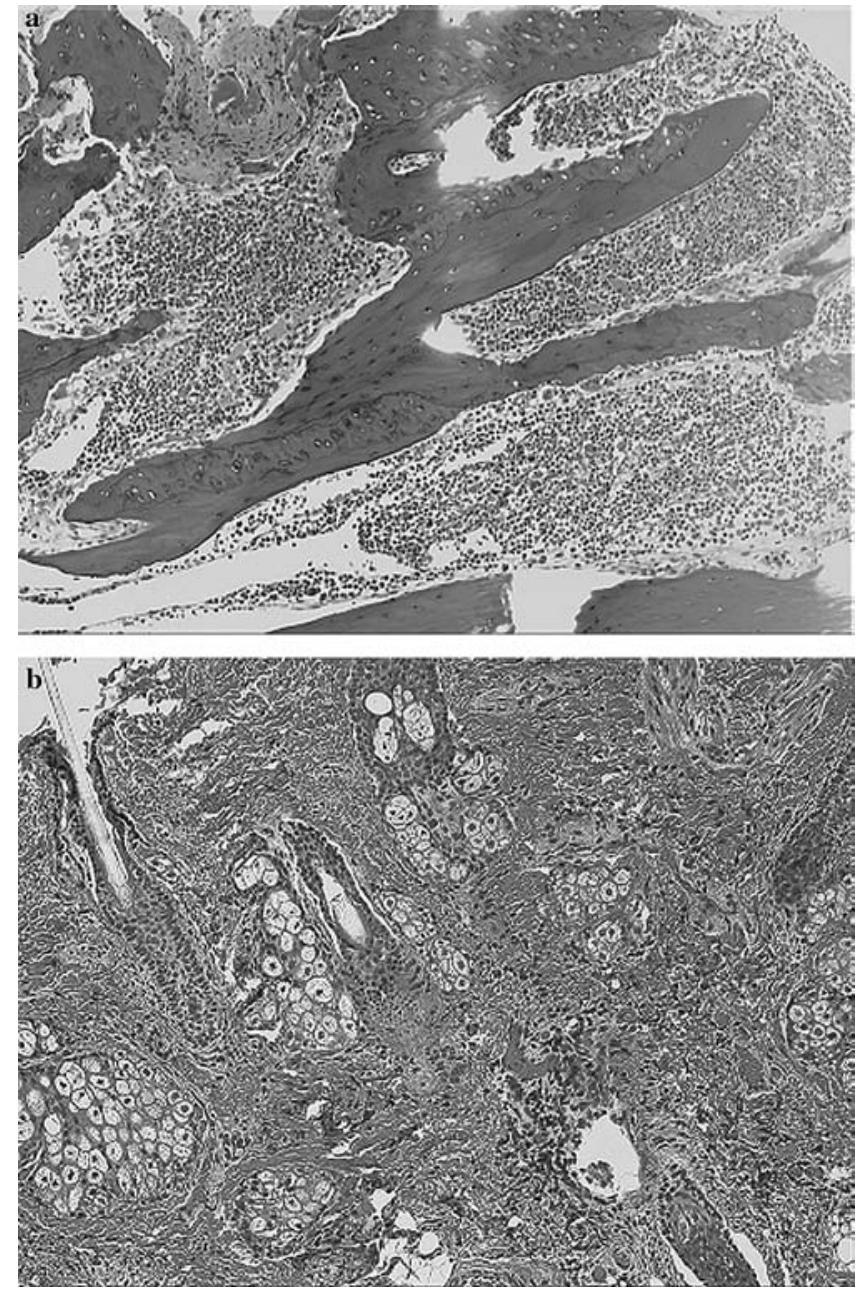

Fig. 3 Histological slices (hematoxylin-eosin staining) of the nasopharyngeal teratoma. a Cartilage and bone with an interspersed stroma are seen. b Stratified squamous epithelium, hair follicles, and sebaceous glands surrounded by adipose tissue are seen

\section{Discussion}

Pituitary duplication is a rare congenital malformation [1-15]. Several articles have described associated mal- 
formations involving the skull base, midline central nervous system structures and the epipharynx. In accordance with the previous reports, in our case all previously reported associated malformations were identified. Until recently the pituitary gland was believed to have a double embryonic origin $[4,8,9,11$, 12]. The anterior lobe of the pituitary gland was believed to arise from the oral ectoderm, and the posterior lobe from the neuroectoderm. According to this embryological theory, a diverticulum (Rathke pouch) originating from the roof of the stomodeum fuses with the downward extending neurohypophyseal bud/diencephalic diverticulum. An early report dating back to 1957 in which Morton [9] studied a case of pituitary duplication questioned this theory. A pituitary duplication cannot be explained in this way. Morton assumed that a duplication of the prochordal plate and the anterior end of the notochordal process at the 15 th-16th day of pregnancy would lead to a duplication of the stomatodaeal region as a whole, and to two areas of persisting contact between neural and surface ectoderms, resulting in two pituitary glands. Mesoderm is then believed to surround and separate the two stomatodaeal areas. In this way, normal structures would form laterally and duplications would occur medially.

Kollias et al. [8] reviewed the literature and support the theory of Morton by stating that notochordal duplication and subsequent prochordal plate duplica- tion is a prerequisite for pituitary gland duplication. The same pathogenetic mechanism is also suggested for other central nervous system malformations, including diastematomyelia. Why a duplication of the prochordal plate and the anterior end of the notochordal process occurs remains unknown. Numerous factors, including maternal exposure to teratogenic factors, have been discussed [8].

Similar to our case, several reports described associated epipharyngeal teratoma in cases of pituitary duplication $[4,8,9,11,12]$. It has consequently been hypothesized that midline inclusion dermoids early in pregnancy could result in a splitting of the pituitary gland primordium [12]. Whether the inclusion of mesenchymal structures, as a precursor of a teratoma, acts as a primary trigger that induces splitting of the notochord or whether the development of a teratoma results from the splitting of the notochord remains unclear. The occurrence of associated skull base and midline malformations in pituitary gland duplication could also indicate a multifactorial etiology.

In our case, ultrasonography excluded associated major abdominal malformations.

Further research focussed on the development of the hypophyseal-hypothalamic axis and the skull base is indicated. Reports of this extremely rare malformation, especially of the imaging findings, could possibly give more insight in the understanding of this complex malformation.

\section{References}

1. Bagherian V, Graham M, Gerson LP, Amstrong DL (1984) Double pituitary glands with partial duplication of facial and brain structures with hydrocephalus. Comput Radiol 8:203-210

2. Bainborogh AR, Hase S (1958) Double hypophysis. Can Med Assoc J 79:912913

3. Bale PM, Reye RD (1976) Epignathus, double pituitary and agenesis of corpus callosum. J Pathol 120:161-164

4. Burke M, Zinkovsky S, Abrantes M, Riley W (2000) Duplication of the hypophysis. Pediatr Neurosurg 33:9599

5. Hamon-Kerautret M, Ares GS, Demondion X, Rouland V, Francke J-P, Pruvo J-P (1998) Duplication of the pituitary gland in a newborn with median cleft face syndrome and nasal teratoma. Pediatr Radiol 28:290-292
6. Hori A (1983) A brain with two hypophyses in median cleft face syndrome. Acta Neuropathol 59:150-154

7. Il'Ina EG, Laziuk GI (1989) A new case of the "double hypophysis-multiple congenital developmental defects" complex. Tsitol Genet 23:45-46

8. Kollias SS, Ball WS, Prenger EC (1995) Review of the embryologic development of the pituitary gland and report of a case hypophyseal duplication detected by MRI. Neuroradiology 37:3-12

9. Morton WRM (1957) Duplication of the pituitary and stomatodaeal structures in a 38-week male infant. Arch Dis Child 32:135-141

10. Roesmann U (1985) Duplication of the pituitary gland and spinal cord. Arch Pathol Lab Med 109:518-520

11. Ryals BD, Brown DC, Levin SW (1993) Duplication of the pituitary gland as shown by MR. AJNR Am J Neuroradiol 14:137-139
12. Shah S, Pereira JK, Becker CJ, Roubal SE (1997) Duplication of pituitary gland. J Comput Assist Tomogr 21:459461

13. Shroff M, Blase S, Jay V, Chitayat D, Armstrong D (2003) Basilar artery duplication associated with pituitary duplication: a new finding. AJNR Am J Neuroradiol 24:956-961

14. Tagliavini F, Pilleri G (1986) Mammillo-hypophyseal duplication (diplomammillo-hypophysis). Acta Neuropathol 69:38-44

15. Uchino A, Sawada A, Takase Y, Fujita I, Kudo S (2002) Extreme fenestration of the basilar artery associated with cleft palate, nasopharyngeal mature teratoma, and hypophyseal duplication. Eur Radiol 12:2087-2090 